\title{
Técnica PRESTO para la revascularización endovascular del segmento femoropoplíteo. Revisión de la literatura
}

\section{PRESTO technique in the endovascular revascularization of the femoro-popliteal segment. Literature review}

\author{
Josefina Duque-Goicochea1*, Paris Martínez-Sosa', Viridiana R. Lara González¹, José I. García-Lugo², \\ Julio A. Serrano-Lozano ${ }^{1}$, Nora Sánchez-Nicolat', Jorge A. Torres-Martínez ${ }^{1}$ y Martín Flores-Escartín ${ }^{1}$ \\ ${ }^{1}$ Departamento de Cirugía, Unidad Hospital Regional “Lic. Adolfo López Mateos", Servicio de Angiología, Cirugía Vascular y Endovascular, Instituto de \\ Seguridad y Servicios Sociales de los Trabajadores del Estado (ISSSTE); "Departamento de Cirugía, Unidad Hospital "Dr. Fernando Quiroz Gutiérrez", \\ Servicio de Cirugía General, Instituto de Seguridad y Servicios Sociales de los Trabajadores del Estado (ISSSTE). Ciudad de México, México
}

\begin{abstract}
Resumen
Se realiza una revisión de las publicaciones médicas acerca de la colocación retrógrada precisa del stent Supera en la arteria femoral superficial (PRESTO) y se notifica un caso. Los términos de búsqueda en inglés fueron PRESTO technique, retrograde access, angioplasty, critical ischemia y superficial femoral artery en las bases de datos Pubmed y Science Direct publicados entre enero de 2017 y diciembre de 2019. Se encontraron cinco informes de casos de pacientes con isquemia crítica tratados mediante técnica PRESTO que incluyeron a pacientes con lesiones en las arterias femoral superficial, poplítea, ilíaca, tronco tibioperoneo y tibial posterior. Se efectuó el acceso retrógrado a través de las arterias poplítea, femoral superficial o tibial posterior. Se registró un índice de permeabilidad al año > 83\%, pero no se observaron fractura del stent Supera ni complicaciones. La colocación de stents mediante acceso retrógrado en el segmento proximal de la arteria femoral superficial puede ser una opción segura, reproducible y con resultados promisorios a corto y largo plazos.
\end{abstract}

Palabras clave: Técnica PRESTO. Acceso retrógrado. Angioplastia. Isquemia crítica. Arteria femoral superficial.

\begin{abstract}
We conducted a review of the literature on the precise retrograde placement of the supera stent in the superficial femoral artery (PRESTO) and a case report. We use the following English search terms: PRESTO technique, retrograde access, angioplasty, critical ischemia, superficial femoral artery, in the Pubmed and ScienceDirect databases published between January 2017 and December 2019. Were found 5 case reports of patients with critical ischemia treated by PRESTO technique, including patients with lesions of superficial femoral artery, popliteal, iliac, tibioperoneal trunk and posterior tibial artery. They were performed with retrograde access through popliteal artery, femoral artery, posterior tibial artery. They reported a patency rate above $83 \%$ at one year, none of them showed a fracture of the Supera stent, none of them presented complications. Retrograde access stenting in the proximal segment of the superficial femoral artery is a safe, reproducible option with adequate short and long-term results.
\end{abstract}

Key words: PRESTO technique. Retrograde access. Angioplasty. Critical limb ischemia. Superficial femoral artery.

Correspondencia:

*Josefina Duque-Goicochea

E-mail: josefinaduque10@gmail.com
Fecha de recepción: 27-11-2020

Fecha de aceptación: 26-01-2021

DOI: 10.24875/RMA.20000045
Available online: $15-04-2021$ Rev Mex Angiol. 2021;49(1):33-40 www.RMAngiologia.com 0377-4740/@ 2021 Sociedad Mexicana de Angiología y Cirugía Vascular y Endovascular, A.C. Publicado por Permanyer. Este es un artículo open access bajo la licencia CC BY-NC-ND license (http://creativecommons.org/licenses/by-nc-nd/4.0/). 


\section{Introducción}

La isquemia crítica que amenaza la extremidad es una etapa avanzada de la enfermedad arterial periférica con notables efectos en la morbilidad y mortalidad $^{1}$. Esta isquemia se caracteriza por la coexistencia de dolor en reposo o ulceración y gangrena del pie o dedos de los pies; se presenta a menudo como una oclusión total crónica (OTC) en el segmento femoropoplíteo (FP).

Las oclusiones de la arteria femoral superficial (AFS) se tratan casi siempre por vía endovascular mediante un acceso anterógrado femoral contralateral, ipsolateral o retrógrado y al final con recanalización intraluminal o subintimal de la luz del vaso. Cuando el acceso anterógrado falla se considera una alternativa válida el acceso retrógrado ${ }^{1-4}$. El 20\% de los intentos de recanalización endovascular por medio de acceso anterógrado falla ${ }^{5,6}$.

La técnica PRESTO (precise retrograde Supera stenting of the ostium) se refiere a la colocación retrógrada precisa del stent Supera ${ }^{\circledR}$ en la AFS. Se trata de una técnica para desplegar de manera segura y precisa el stent Supera ${ }^{\circledR}$ en el ostium de la arteria sin comprometer la luz de las arterias femoral profunda y femoral común $^{1,7}$. La técnica recomienda una meticulosa preparación del vaso mediante predilatación sistemática con balones, de forma secuencial y progresiva. Este protocolo garantiza que la luz arterial tenga un tamaño suficiente para el adecuado acoplamiento a la geometría del stent, de tal modo que se evite el alargamiento o la compresión de éste. Los accesos retrógrados para el tratamiento de una OTC en la AFS ha producido resultados exitosos, en términos de la permeabilidad a largo plazo en diversas series de casos $^{8}$. Además, el uso de stents de nitinol en lesiones largas de la AFS se ha relacionado con bajas tasas de fractura y reestenosis ${ }^{9}$. El uso del stent Supera ${ }^{\circledR}$ (Abbott Vascular, Santa Clara, California) en la AFS se acompaña de un éxito técnico del $100 \%$. La permeabilidad primaria registrada es del $94.1 \%$ a un año y se obtuvo mejoría clínica en la mayoría de los pacientes ${ }^{10}$.

La angioplastia también puede reducir el riesgo de ruptura arterial. El acceso retrógrado y el despliegue inverso del stent Supera ${ }^{\circledR}$ permiten al operador mayor precisión y seguridad, ya que colocar la primera corona del stent justo en el ostium de la AFS puede considerarse desafiante cuando se realiza de forma anterógrada.

Las fracturas del stent se vinculan con reestenosis o reoclusión ${ }^{11,12}$; asimismo, los pacientes con diabetes mellitus tienen resultados morfológicos muy pobres después de la colocación de un stent en la AFS de largo segmento (15 de 24 con reestenosis, todas difusas; $78 \%$ riesgo de estenosis). La hiperglucemia acentúa los estragos de la hipertrofia en una herida en respuesta al traumatismo de la pared del vaso después de la implantación del dispositivo, lo que da lugar a una obstrucción neointimal ${ }^{13,14}$. Es por ello que los pacientes tratados deben someterse a un estricto control glucémico.

El objetivo de este artículo es revisar las publicaciones médicas actuales de esta técnica y notificar un caso realizado en el servicio de los autores. Se efectuó una revisión de las publicaciones mediante los siguientes términos de búsqueda en inglés: PRESTO technique, retrograde access, angioplasty, critical ischemia, superficial femoral artery en las bases de datos Pubmed y Science Direct publicados entre enero de 2017 y diciembre de 2019.

\section{Informe del caso}

Paciente masculino de 65 años con diabetes mellitus de tipo 2 de larga evolución y antecedentes de tabaquismo desde los 16 años con índice tabáquico de 16. Se había sometido a amputación transfemoral de miembro pélvico izquierdo dos años antes, secundaria a isquemia crítica WIFI IV. Acudió a valoración por lesión isquémica de más de cuatro meses de evolución en el maléolo medial del miembro pélvico derecho. La exploración física reveló pulso femoral de grado 2/2, poplíteo de grado $2 / 2$, tibial posterior de $1 / 2$, pulso pedio ausente, flujo bifásico en arteria tibial anterior y posterior, presión arterial sistólica en tobillo de $180 \mathrm{mmHg}$, flujo bifásico en arteria pedia, pero el índice tobillo-brazo (ITB) no fue compresible. En el maléolo interno presentaba una úlcera de bordes regulares de $4 \mathrm{~cm}$ de diámetro con profundidad de $3 \mathrm{~mm}$, sin exudado, de fondo limpio; la segunda úlcera se encontraba en la cara posterior de la pierna a nivel de tercio inferior de ésta, con bordes regulares y dimensión de $3 \times 2 \mathrm{~cm}$ con profundidad de $1 \mathrm{~mm}$ sin exudado y con exposición tendinosa. Se solicitó un ultrasonido Doppler de la arteria femoral común (AFC) que reveló flujos trifásicos, con velocidad de pico sistólico (VPS) de $122 \mathrm{~cm} / \mathrm{s}$, velocidad de pico diastólico (VPD) de $2.7 \mathrm{~cm} / \mathrm{s}$ e índice de resistencia (IR) de 0.98 en AFS con datos de estenosis (Fig. 1) desde el ostium y con recanalización distal en la arteria poplítea; a este nivel se identificaron flujos bifásicos con VPS de $91.7 \mathrm{~cm} / \mathrm{s}$, VPD de $6.1 \mathrm{~cm} / \mathrm{s}$ e IR de 0.95 , con flujos bifásicos desde ese nivel. Con estos datos se estadificó como WIFI (wound 1, ischemia 3, 


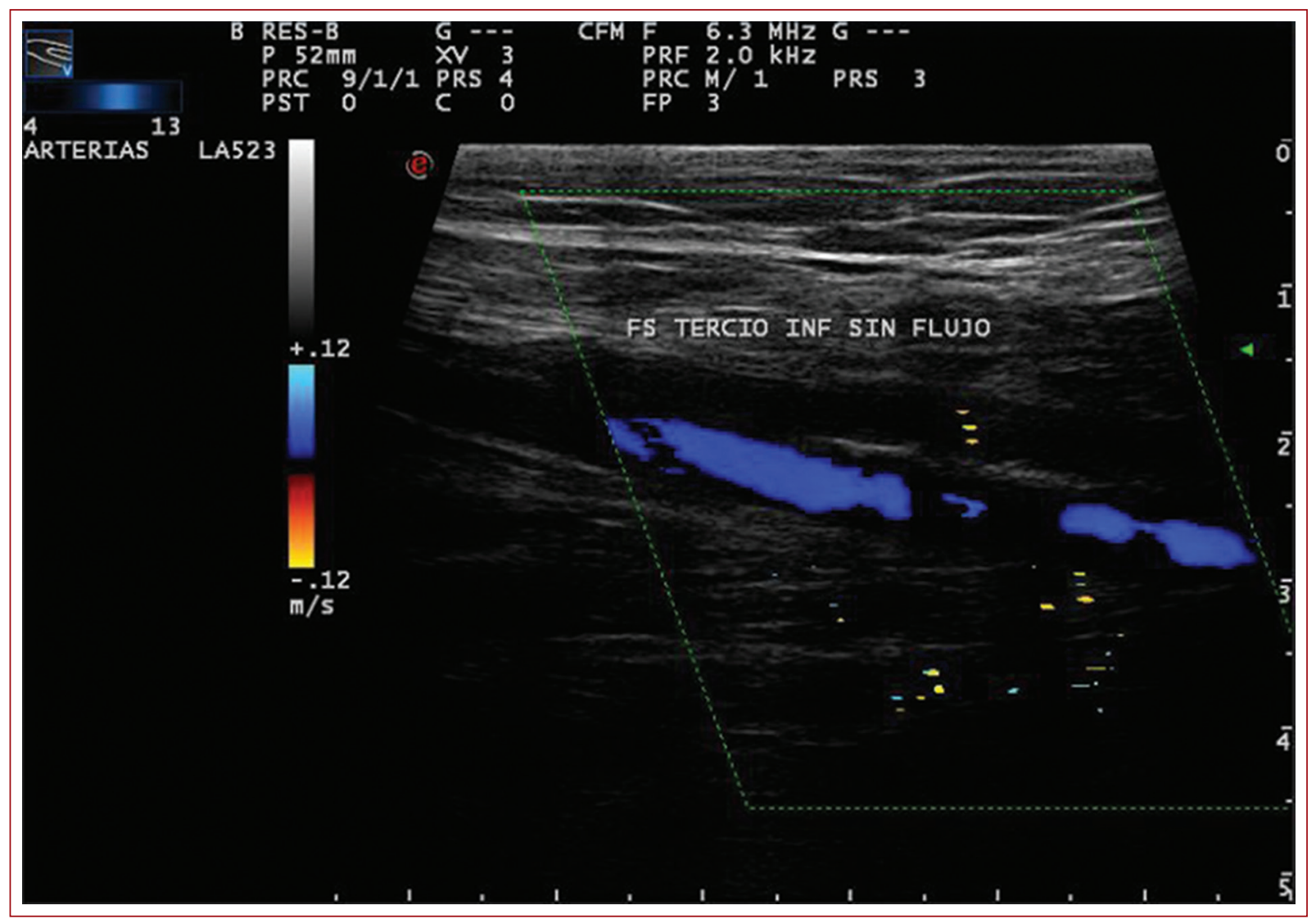

Figura 1. Ultrasonido Doppler preoperatorio que muestra OTC en AFS.

foot infection 0) estadio 3 y enfermedad arterial periférica femoropoplítea TASC D. Se hospitalizó, se obtuvieron estudios de laboratorios con informe de creatinina de $1.59 \mathrm{mg} / \mathrm{dl}$, BUN de $26 \mathrm{mg} / \mathrm{dl}$, urea de $55.3 \mathrm{mg} / \mathrm{dl}$, y se calculó una tasa de filtrado glomerular con la ecuación modificada del estudio MDRD (Modification of Diet in Renal Disease) de $34.76 \mathrm{ml} / \mathrm{min} / \mathrm{m}^{2}$; el servicio de nefrología lo valoró y no contraindicó el procedimiento y calculó riesgo de nefropatía de $14 \%$ con riesgo de diálisis de $0.12 \%$; se consideró elegible para arteriografía diagnóstica. Una vez en la sala de hemodinamia, con el paciente en decúbito dorsal, se practicó una punción eco guiada a nivel de la arteria femoral común derecha y se realizó arteriografía diagnóstica que identificó OTC $>20 \mathrm{~cm}$ desde el ostium de la arteria femoral superficial hasta su desembocadura en la arteria poplítea, que se clasificó como GLASS estadio II; se introdujo guía hidrofílica 0.35 " y se intentó realizar el cruce de la oclusión crónica total de la arteria femoral superficial mediante abordaje anterógrado. Tras fracasar se decidió un abordaje retrógrado a través de la arteria tibial anterior: se cruzó dicha oclusión y se efectuó una angioplastia de la arteria femoral superficial exitosa, pero con algunos segmentos $<1 \mathrm{~cm}$ con estenosis residual $>30 \%$, por lo que se decidió colocar un stent Supera ${ }^{\circledR}$ retrógrado con la técnica PRESTO.

\section{Técnica}

Se infiltra anestesia local en la región inguinal derecha y se realiza una punción eco guiada de la arteria femoral común derecha; se coloca un introductor $6 \mathrm{Fr}$ y se administra un bolo de heparina intraarterial; se lleva a cabo la arteriografía diagnóstica, se observa una oclusión total de la arteria femoral superficial desde el ostium y se intenta cruzar la oclusión en múltiples ocasiones con guía hidrofílica 0.35 " y teflonada 0.35 " sin lograr cruzar la oclusión; se decidió utilizar un acceso retrógrado. Se infiltra lidocaína en la región anterior de tercio medio de la pierna y se realiza punción eco guiada en el tercio distal de la arteria tibial anterior con instrumentos de micropunción; se obtiene arteriografía para confirmar la localización intraluminal de los instrumentos y se introduce una guía 0.14 " x 300 


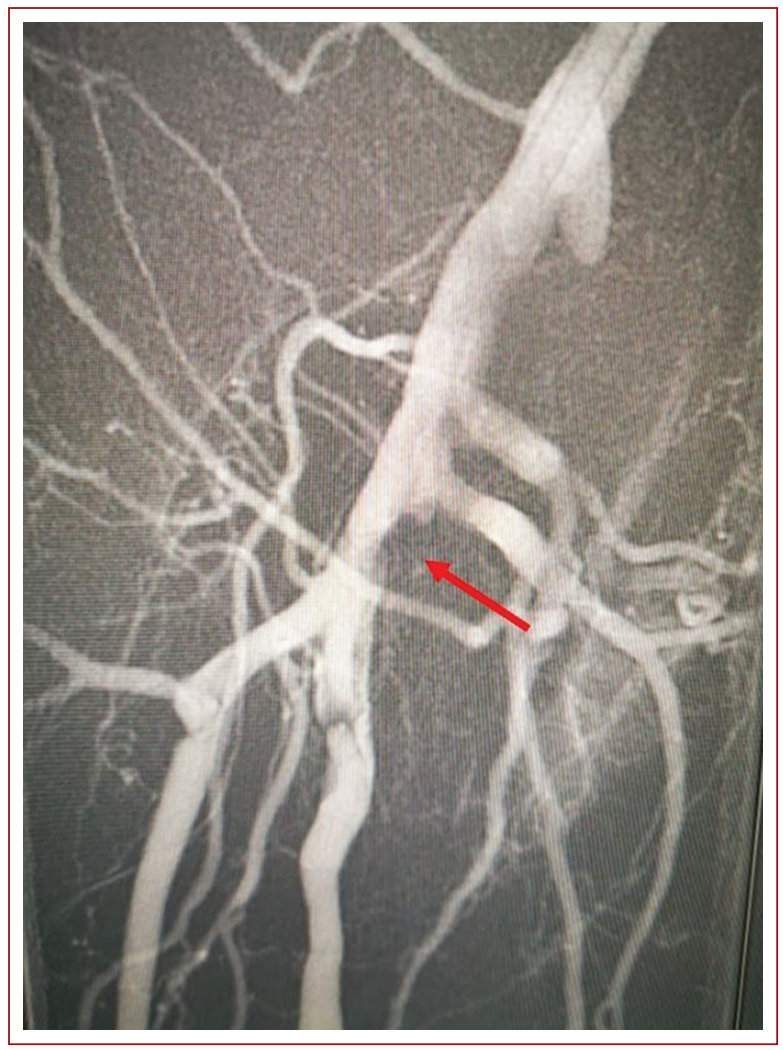

Figura 2. Arteriografía de una estenosis en AFS.

cm y catéter de soporte CXI (Cook Medical, Bloomington) hasta alcanzar el sitio de la oclusión total de la AFS, la cual se cruza y se practica la angioplastia con balón Armada $^{\circledR}$ de $1.5 \mathrm{~mm}$ x $120 \mathrm{~cm}$ (Abbott, Santa Clara, California) por dos minutos, luego balón Freeway ${ }^{\circledR}$ de $2 \mathrm{~mm} \times 120 \mathrm{~cm}$ (Eurocor, Bonn, Alemania) por dos minutos, después balón Armada ${ }^{\circledR}$ de $4 \mathrm{~mm} x$ $200 \mathrm{~cm}$ (Abbott, Santa Clara, California) por dos minutos y al final balón Armada ${ }^{\circledR}$ de $6 \mathrm{~mm} \times 200 \mathrm{~cm}$ (Abbott ${ }^{\circledR}$, Santa Clara, California) por dos minutos más. La arteriografía de control delinea estenosis residual $>30 \%$ y se decide la colocación de stent Supera ${ }^{\circledR}$ (Abbott Vascular, Santa Clara, California) en el ostium de la AFS de $6.5 \times 150 \mathrm{~mm}$ y en el tercio medio y distal un stent Supera ${ }^{\circledR}$ de $5.5 \times 100$; la arteriografía de control muestra un adecuado paso del medio de contraste en todo el trayecto de la AFS, por lo que se decide finalizar el procedimiento (Fig. 2 y 3 ).

\section{Seguimiento del paciente}

El paciente ha recibido seguimiento en la consulta externa de angiología durante nueve meses. El

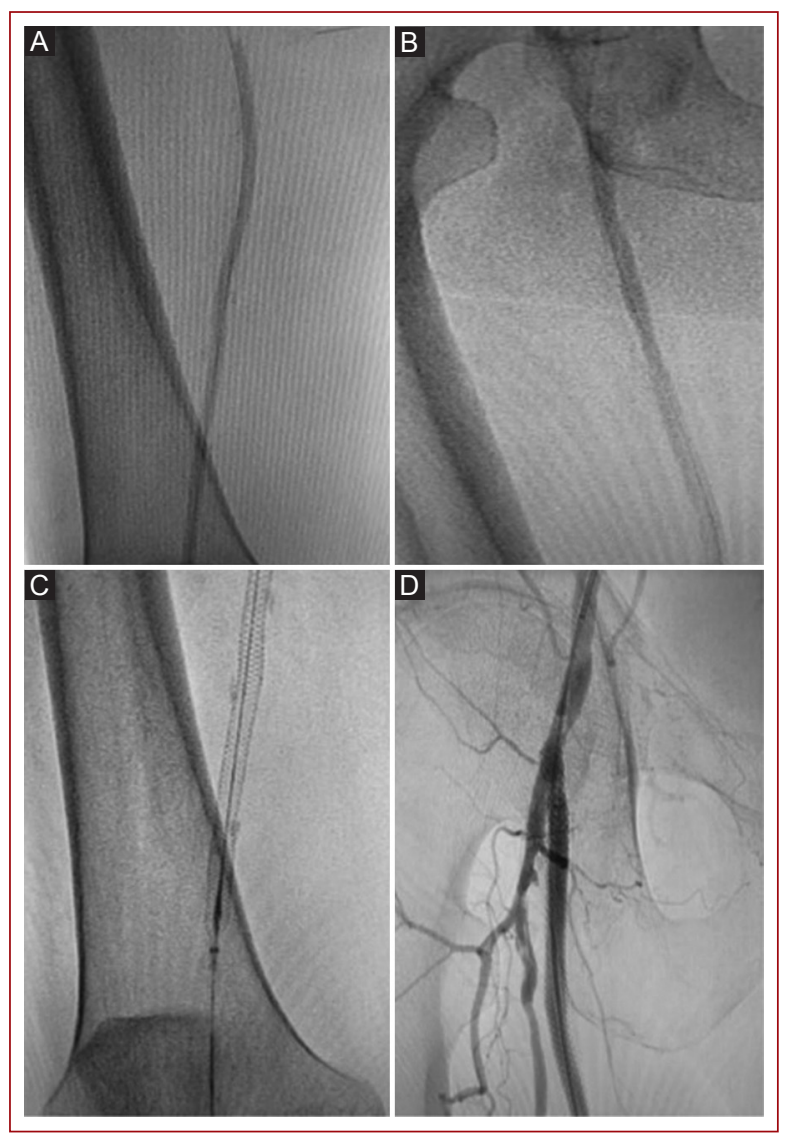

Figura 3. Colocación de stent mediante la técnica PRESTO. A: Predilataciones progresivas con balón de la AFS. B: Arteriografía de control de AFS. C: Liberación del stent mediante la técnica PRESTO. D: Arteriografía de control que muestra áreas de estenosis con adecuada permeabilidad del stent.

paciente no sufre dolor en la extremidad, las úlceras tienen tejido de granulación del $80 \%$ y, en cuanto a su tamaño, la úlcera de la cara interna a nivel maleolar redujo su diámetro en un $75 \%$, ahora con $1 \mathrm{~cm}$ de diámetro y profundidad de $1 \mathrm{~mm}$ y $\sin$ exudado. La segunda úlcera, en la cara posterior de la pierna en el tercio distal, redujo su tamaño un $67 \%$, con dimensiones de $2 \times 1 \mathrm{~cm}$, y $1 \mathrm{~mm}$ de profundidad sin exudado. No hay datos de infección ni exposición tendinosa. Se indicó antes del procedimiento tratamiento con ácido acetilsalicílico (150 mg/24 h); durante el tratamiento se administraron 5,000 UI de heparina no fraccionada y luego del tratamiento se indicó tratamiento anticoagulante con rivaroxabán a dosis de $20 \mathrm{mg}$ cada 24 horas. Se realizó un ultrasonido Doppler de control a los tres meses del procedimiento en el que se observó permeabilidad del stent en la AFS, con VPS de $57.0 \mathrm{~cm} / \mathrm{s}$, 
Tabla 1. Publicaciones sobre la liberación del stent retrógrado

\begin{tabular}{|c|c|c|c|c|c|c|}
\hline $\begin{array}{l}\text { Primer autor, año, } \\
\text { referencia }\end{array}$ & $\begin{array}{l}\text { Número de } \\
\text { casos } \\
\text { informados } \\
\text { por estudio }\end{array}$ & Comorbilidades & $\begin{array}{l}\text { TASC/segmentos } \\
\text { afectados }\end{array}$ & Tratamiento endovascular & Tratamiento post-PRESTO & Permeabilidad \\
\hline $\begin{array}{l}\text { Luis M. Palena, } \\
2018 \text {, } \\
\text { Journal of } \\
\text { Endovascular } \\
\text { Therapy }{ }^{1}\end{array}$ & 21 & No se mencionan & No se mencionan & $\begin{array}{l}10 \text { Acceso retrógrado por arteria poplítea } 0 \text { arteria } \\
\text { tibial anterior (ATA) } \\
\text { Colocación de stent Supera }{ }^{\circledR} \text { : } \\
6 \text { desde ATA proximal. } \\
2 \text { desde arteria tibial posterior } \\
2 \text { desde una punción retrógrada de un stent en el } \\
\text { medio de la } \\
\text { AFS distal } \\
6 \text { de la AFS distal nativa/proximal poplítea } \\
5 \text { desde ATA distal }\end{array}$ & $\begin{array}{l}\text { Antes del tratamiento } \\
\text { endovascular, } \\
\text { ácido } \\
\text { acetilsalicílico ( } 81-325 \\
\mathrm{mg} \text { y y ticlopidina }(500 \mathrm{mg} \text { ) } \\
\text { o clopidogrel } \\
\text { (300-600 mg) } \\
\text { Durante el procedimiento }\end{array}$ & $\begin{array}{l}\text { A } 9 \text { meses no se } \\
\text { ha observado } \\
\text { fractura del stent } \\
\text { Supera }{ }^{\circledR} 0 \\
\text { pérdida de la } \\
\text { permeabilidad }\end{array}$ \\
\hline $\begin{array}{l}\text { Daniel Raskin, } \\
2019, \\
\text { J Vasc Interv } \\
\text { Radiol }^{2}\end{array}$ & 12 & $\begin{array}{l}\text { Hipertensión } \\
\text { Obesidad } \\
\text { Diabetes } \\
\text { Enfermedad } \\
\text { cardiovascular } \\
\text { Tabaquismo } \\
\text { Aneurisma } \\
\text { abdominal aórtico } \\
\text { Insuficiencia renal } \\
\text { crónica }\end{array}$ & $\begin{array}{l}12 \text { TASC D } \\
8 \text { Femoropoplítea por } \\
\text { encima de la rodilla } \\
4 \text { Femoropoplítea por } \\
\text { arriba y debajo de la rodilla } \\
1 \text { Ilíaca y femoropoplítea } \\
\text { por encima de la rodilla } \\
1 \text { Femoropoplítea por } \\
\text { arriba } \\
\text { y debajo de la rodilla } \\
1 \text { Injerto femoropoplíteo } \\
\text { ocluido }\end{array}$ & $\begin{array}{l}\text { Todos los accesos fueron a través de la arteria pedia } \\
13 \text { Stents }\end{array}$ & $\begin{array}{l}\text { Los pacientes sometidos } \\
\text { a la colocación de un } \\
\text { stent recibieron } \\
\text { un régimen antiplaquetario } \\
\text { de } 75 \mathrm{mg} \text { de clopidogrel y } \\
81-325 \mathrm{mg} \text { de } \\
\text { ácido acetilsalićlico diario } \\
\text { durante al menos } 3 \text { meses. } \\
\text { Los pacientes sometidos } \\
\text { a una angioplastia simple } \\
\text { recibieron ácido } \\
\text { acetilsalicílico }\end{array}$ & $\begin{array}{l}\text { Seguimiento } \\
\text { promedio de } 7.1 \\
\text { meses } \\
\text { Índice de } \\
\text { rescate de } \\
\text { extremidad a un } \\
\text { año de } 83 \% \text {. }\end{array}$ \\
\hline $\begin{array}{l}\text { Lorenzo Patrone, } \\
2019, \\
\text { CVIR } \\
\text { Endovascular }\end{array}$ & 1 & $\begin{array}{l}\text { Oclusión de } 1 \\
\text { derivación proximal } \\
\text { femoropoplítea y } 2 \\
\text { femoropoplíteas } \\
\text { ocluidas distales a } \\
\text { la ATA y TTP }\end{array}$ & $\begin{array}{l}\text { OTC en AFS } \\
\text { APOP y ATP } \\
\text { Oclusión de APOP, ATP y } \\
\text { ATA }\end{array}$ & $\begin{array}{l}\text { Acceso por ATA } \\
\text { En ATA se colocó stent medicado BTK XIENCE Prime } \\
\text { de } 3.5 \mathrm{~mm} \text { (Abbott, Chicago, Illinois) } \\
\text { Después de la angioplastia subintimal de la AFS, APOP } \\
\text { y ATA proximal, } \\
\text { se implantó un XIENCE Prime de } 4 \mathrm{~mm} \text { (Abbott, } \\
\text { Chicago, Illinois). } \\
\text { La APOP y AFS también requerín la colocación de } \\
\text { stents, realizada mediante la superposición de } \\
\text { Supera }{ }^{\circledR} \text { de } 5.5 \mathrm{~mm} \text { después de la angioplastia } \\
\text { subintimal de la AFS, APOP y proximal } \\
\text { ATA, este último implantado por un XIENCE Prime de } 4 \mathrm{~mm} \text {. } \\
\text { BTK Drug Eluting Stent (Abbott, Chicago, Illinois). } \\
\text { La APOP y AFS también requerían la colocación de } \\
\text { stents, realizada mediante la superposición de } \\
\text { stent Supera }{ }^{\circledR} \text { de } 5.5 \mathrm{~mm} \text { (Abbott, Chicago, Illinois) y a } \\
\text { nivel de AFS proximal se colocó uno de } 6 \text { mm Pro stent } \\
\text { absoluto (Abbott, Chicago, Illinois). }\end{array}$ & No se menciona & $\begin{array}{l}\text { Se observó } \\
\text { permeabilidad de } \\
\text { los stents por US } \\
\text { Doppler a } 3,6,12 \\
\text { y } 24 \text { meses de } \\
\text { seguimiento; se } \\
\text { informó } \\
\text { flujo monofásico } \\
\text { a nivel de } \\
\text { DP (velocidad, } 70 \\
\text { cm/s). }\end{array}$ \\
\hline
\end{tabular}


Tabla 1. Publicaciones sobre la liberación del stent retrógrado (Continuación)

\begin{tabular}{|c|c|c|c|c|c|c|}
\hline $\begin{array}{l}\text { Primer autor, año, } \\
\text { referencia }\end{array}$ & $\begin{array}{l}\text { Número de } \\
\text { casos } \\
\text { informados } \\
\text { por estudio }\end{array}$ & Comorbilidades & $\begin{array}{l}\text { TASC/segmentos } \\
\text { afectados }\end{array}$ & Tratamiento endovascular & Tratamiento post-PRESTO & Permeabilidad \\
\hline $\begin{array}{l}\text { Mert Dumantepe, } \\
2017, \\
\text { Vascular and } \\
\text { Endovascular } \\
\text { Surgery }{ }^{4}\end{array}$ & 28 & $\begin{array}{l}22 \text { Hipertensión } \\
16 \text { Diabetes } \\
21 \text { Fumadores } \\
11 \text { Enfermedad } \\
\text { coronaria } \\
6 \text { Enfermedad } \\
\text { carotídea } \\
13 \\
\text { Hiperlipoproteinemia }\end{array}$ & $\begin{array}{l}7 \text { TASC II C } \\
21 \text { TASC II D }\end{array}$ & $\begin{array}{l}\text { Sitio de acceso retrógrado: } \\
26 \text { Arteria poplítea } \\
2 \text { Espacio subintimal } \\
\text { Se colocó stent Supera } \\
\text { Se utilizó el dispositivo Rotarex (Straub Medical, } \\
\text { Wangs, Suiza) } \\
\text { Todas las lesiones se dilataron antes de la colocación } \\
\text { del stent } \\
\text { Tratamiento de inflow } \\
4 \text { Angioplastia simple } \\
3 \text { Angioplastia con balón medicado } \\
\text { outflow } \\
3 \text { Angioplastia simple } \\
6 \text { Angioplastia con balón medicado } \\
1 \text { Stent liberador de fármaco }\end{array}$ & $\begin{array}{l}\text { ASA + clopidogrel } \\
\text { durante } 30 \text { días, luego } \\
\text { sólo ASA }\end{array}$ & $\begin{array}{l}\text { IP: } 1 \text { mes } 100 \% \text {, } \\
6 \text { meses } 92.8 \% \text { y } \\
1 \text { año } 85.7 \% \text {. } \\
3 \text { reestenosis } \\
1 \text { OTC }\end{array}$ \\
\hline $\begin{array}{l}\text { Junya Matsumi, } \\
\text { 2017, } \\
\text { Ann Vasc Surg }\end{array}$ & $\begin{array}{l}20 \text { pacientes } \\
21 \\
\text { extremidades }\end{array}$ & $\begin{array}{l}3 \text { Hemodiálisis } \\
11 \text { DM } \\
16 \text { hipertensión } \\
16 \\
\text { Hipercolesterolemia } \\
14 \text { Tabaquismo } \\
\text { previo suspendido } \\
6 \text { Tabaquismo activo } \\
4 \text { Enfermedad } \\
\text { cerebrovascular } \\
2 \text { Infarto al } \\
\text { miocardio } \\
2 \text { Cirugía de } \\
\text { derivación de arteria } \\
\text { coronaria con } \\
\text { colocación de } \\
\text { injerto } \\
1 \text { Isquemia crítica }\end{array}$ & $\begin{array}{l}7 \text { TASC II B } \\
3 \text { TASC II C } \\
11 \text { TASC II D } \\
21 \text { OTC } \\
18 \text { AFS } \\
1 \text { APOP, P2 } \\
2 \text { Al }\end{array}$ & $\begin{array}{l}\text { Sitio de acceso retrógrado: } \\
16 \text { APOP }(76.2 \%) \\
2 \text { AFC }(9.5 \%) \\
2 \text { AFS distal }(9.5 \%) \\
1 \text { ATP }(4.8 \%) \\
16 \text { Uso de IVUS } \\
19 \text { Uso de stent }(94.5 \%) \\
2 \text { Angioplastia con balón }(5.5 \%) \\
10 \text { Punción ipsolateral }(47.6) \\
11 \text { Punción contralateral crossover }(52.4 \%) \\
\text { Número de stents implantados: } \\
11 \text { Smart }(26.1 \%) \\
2 \text { Zilver } 518(4.8 \%) \\
9 \text { Zilver PTX } 9(21.4 \%) \\
2 \text { Life }(4.8 \%) \\
18 \text { Misago }(42.9 \%)\end{array}$ & $\begin{array}{l}12 \text { Cilostazol } \\
16 \text { Clopidogrel } \\
20 \text { Ácido acetilsalicílico } \\
16 \text { Estatina }\end{array}$ & $\begin{array}{l}\text { IP: } 1 \text { año } 89.5 \% \text {, } \\
2 \text { años } 72 \%, 3 \\
\text { años } 41.2 \%\end{array}$ \\
\hline
\end{tabular}




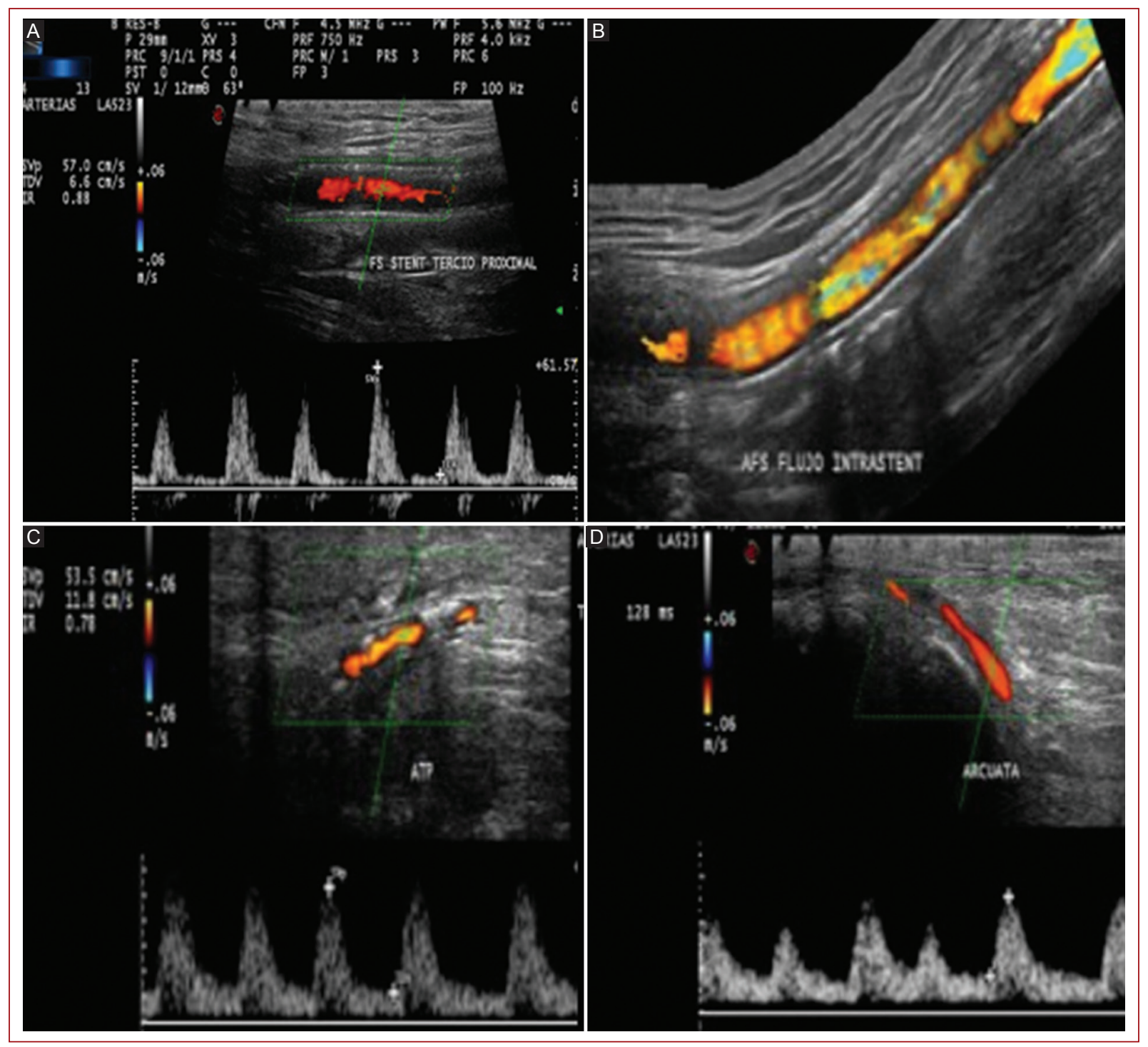

Figura 4. USG de control tres meses después del procedimiento. A: Stent permeable en AFS con flujo trifásico. B: AFS permeable. C: ATP permeable con flujo bifásico. D: Arteria arqueada con tiempo de aceleración de clase II.

TDV de $6.6 \mathrm{~cm} / \mathrm{s}$ e IR de 0.88 en ATP, con VPS de $53.5 \mathrm{~cm} / \mathrm{s}$, TDV de $11.8 \mathrm{~cm} / \mathrm{s}$ e IR de 0.78 en la arteria arqueada con un tiempo de aceleración de $128 \mathrm{~ms}$ (Fig. 4).

\section{Discusión}

La reapertura endovascular de las OTC en la AFS es un desafío técnico; se necesita una larga curva de aprendizaje y conocimiento de muchos dispositivos especiales y de recanalización con diferentes técnicas, como el cruce subintimal, abordaje transcolateral 0 accesos retrógrados ${ }^{2}$. Estos últimos constituyen una técnica para abordar oclusiones totales crónicas de la
AFS después de una recanalización anterógrada falli$\mathrm{da}^{3}$. El acceso retrógrado es una opción en el caso de lesiones proximales complejas, ya que hasta en el $20 \%$ de las ocasiones no es posible un acceso anterógra$\mathrm{do}^{8}$. En el caso notificado no fue posible realizar una punción en la arteria femoral común contralateral debido a que la AFC de la pierna contralateral tenía una placa ateromatosa en la cara anterior de $65 \%$.

Cuando se decide colocar un stent vasculomimético es imprescindible el principio de una adecuada preparación del vaso, ya sea con angioplastia o aterectomía; en caso de lesiones extensas con estenosis residuales y calcificadas se recomienda un stent para mejorar los índices de permeabilidad ${ }^{1}$. De acuerdo con las 
publicaciones médicas, los stents se colocaron en la arteria femoral o la arteria poplítea por vía retrógrada (a través de las arterias poplítea, tibial anterior, tibial posterior o pedia), con tasas de permeabilidad primaria $>80 \%$ a un año. Del mismo modo, luego de nueve meses (en el caso notificado) se mantiene permeable el stent y con valores hemodinámicos aceptables.

En las publicaciones médicas hay pocos informes de casos del tratamiento endovascular de OTC de la AFS mediante el acceso retrógrado con colocación de stent con técnica PRESTO, dado que es un procedimiento relativamente reciente. Sin embargo, en las publicaciones revisadas se reconocieron resultados adecuados en la permeabilidad de los stents colocados mediante esta técnica, sin fractura de éstos y con índices de permeabilidad $>80 \%$ a un año, lo que hace pensar que es una técnica segura y con aceptables índices de permeabilidad (Tabla 1). No obstante, en los casos informados no se cuenta con seguimientos mayores de un año.

\section{Conclusión}

El uso de la técnica PRESTO para el tratamiento de la OTC en la AFS ha permitido obtener resultados exitosos con adecuadas tasas de permeabilidad a un año. La colocación de stents mediante acceso retrógrado en el segmento proximal de la arteria femoral superficial es una opción segura, que puede realizarse en pacientes con isquemia crítica y riesgo quirúrgico alto. Además, es una técnica reproducible.

\section{Agradecimientos}

Los autores agradecen a las autoridades del Hospital Regional Lic. Adolfo López Mateos (ISSSTE), a los médicos adscritos y personal que conforma el servicio de Angiología y Cirugía Vascular y Endovascular, por las facilidades brindadas para la realización de este trabajo.

\section{Conflicto de intereses}

Los autores declaran que no tienen conflicto de intereses.

\section{Financiamiento}

Esta investigación no ha recibido ninguna beca específica de instituciones de los sectores públicos, comercial, o sin ánimo de lucro.

\section{Responsabilidades éticas}

Protección de personas y animales. Los autores declaran que para esta investigación no se han realizado experimentos en seres humanos ni en animales.

Confidencialidad de los datos. Los autores declaran que han seguido los protocolos de su centro de trabajo sobre la publicación de datos de pacientes.

Derecho a la privacidad y consentimiento informado. Los autores han obtenido el consentimiento informado del paciente referido en el artículo.

\section{Bibliografía}

1. Palena LM, Díaz-Sandoval LJ, Raja LM, Morelli L, Manzi M. Precise retrograde Supera stenting of the ostium (presto) of the superficial femoral artery for complex femoropoplítea occlusions: The PRESTO Technique. J Endovasc Ther. 2018;25(5):588-591. doi: 10.1177/ 1526602817753388. Epub 2018 Jan 24. PMID: 29363382.

2. Raskin D, Khaitovich B, Silverberg D, Halak M, Balan S, Rimon U. Primary retrograde dorsalis pedis artery single access for revascularization of chronic total occlusion in patients with critical limb ischemia. J Vasc Interv Radiol. 2019;30(4):554-559. doi: 10.1016/j.jvir.2018.08.030. Epub 2019 Mar 1. PMID: 30827752

3. Patrone L, Stehno O. Retrograde insertion of the outback reentry device from a tibial artery for complex infrainguinal recanalization CVIR. Endovascular. 2019;30;2(1):47. doi: 10.1186/s42155-019-0088-7. PMID 32026118; PMCID: PMC6966385

4. Dumantepe M. Retrograde popliteal access to percutaneous peripheral intervention for chronic total occlusion of superficial femoral arteries. Vascular Endovascular Surgery. 2017;51(5):240-246. doi: 10.1177/ 1538574417698902. Epub 2017 Jun 8. PMID: 28595481

5. Ruzsa Z, Nemes B, Bánsághi Z, Tóth K, Kuti F, Kudrnova S, Transpedal Access after failed anterograde recanalization of complex below-the-knee and femoropoliteal occlusions in critical limb ischemia. Catheterization and Cardiovascular Interventions. 2014,;83:997-1007.

6. Schmidt A, Bausback Y, Piorkowski M, Werner M, Bräunlich S, Ulrich M, et al. Retrograde recanalization technique for use after failed antegrade angioplasty in chronic femoral artery occlusions. J Endovasc Ther. 2012;19:23-29.

7. Yamawaki M, Hirano K, Nakano M, Sakamoto Y, Takimura H, Araki M, et al. Deployment of self-expandable stent for complex proximal superficial femoral artery lesions involving the femoral bifurcation with or without jailed deep femoral artery. Catheter Cardiovasc Interv. 2013;81(6):10311041. doi: 10.1002/ccd.24502. Epub 2012 Nov 14. PMID: 22639451.

8. Matsumi J, Takada T, Moriyama N, Ochiai T, Tobita K, Shishido K, et al. Initial and long-term results of a microcatheter-based retrograde approach for the endovascular treatment of chronic total occlusion in iliac or femoropopliteal arteries. Ann Vasc Surg. 2017;41:176-185. doi: 10.1016/j. avsg.2016.08.047. Epub 2017 Feb 24. PMID: 28238927.

9. Bosiers M, Torsello G, Gissler HM, Ruef J, Müller-Hülsbeck S, Jahnke T, et al. Nitinol stent implantation in long superficial femoral artery lesions: 12-month results of the DURABILITY I study. J Endovasc Ther. 2009;16:261-269. doi: 10.1583/08-2676.1. PMID: 19642788.

10. Palena LM, Díaz-Sandoval LJ, Sultato E, Bigrato C, Candeo A, Brocco E, et al. Feasibility and 1 year outcomes of Subintimal revascularization with Supera stenting of long femoropopliteal occlusions in critical limb ischemia. The "Supersub" study. Catheter Cardiovasc Interv. 2017;89(5):910920. doi: 10.1002/ccd.26863. Epub 2016 Nov 12. PMID: 27862880.

11. Laird JR, Katzen BT, Scheinert D, Lammer J, Carpenter J, Buchbinder M, et al. Nitinol stent implantation vs. balloon angioplasty for lesions in the superficial femoral and proximal popliteal arteries of patients with claudication: three-year follow-up from the RESILIENT randomized trial. J Endovasc Ther. 2012;19(1):1-9. doi: 10.1583/11-3627.1. PMID: 22313193.

12. Scheinert D, Grummt L, Piorkowski M, Sax J, Schneiter S, Ulrich M et al. A novel selfexpanding interwoven nitinol stent for complex femoropopliteal lesions: 24-month results of the SUPERA SFA registry. J Endovasc Ther. 2011;18:745-752.

13. Sabeti S, Mlekusch W, Amighi J, Minar E, Schillinger M. Primary patency of long-segment self-expanding nitinol stents in the femoropopliteal arteries. J Endovasc Ther. 2005;12(1):6-12. doi: 10.1583/04-1359.1. PMID: 15683273.

14. Manzi M, Palena LM, Cester G. Endovascular techniques for limb salvage in diabetics with crural and pedal disease. J Cardiovasc Surg (Torino). 2011;52(4):485-92. PMID: 21792156 